\title{
Ad Hoc On-Demand Multipath Distance Vector Routing Protocol Based on Node State
}

\author{
Jieying Zhou, Heng Xu, Zhaodong Qin, Yanhao Peng, Chun Lei \\ School of Information Science and Technology, Sun Yat-sen University, Guangzhou, China \\ Email: isszjy@mail.sysu.edu.cn
}

Received June 2013

\begin{abstract}
To improve the performance of Ad hoc on-demand multipath distance vector (AOMDV) protocol, we proposed NSAOMDV which is short for "AOMDV based on node state". In NS-AOMDV, we introduce node state to improve AOMDV's performance in selecting main path. In route discovery process, the routing update rule calculates the node weight of each path and sorts the path weight by descending value in route list, and we choose the path which has the largest path weight for data transmission. NS-AOMDV also uses the technology of route request (RREQ) packet delay forwarding and energy threshold to ease network congestion, limit the RREQ broadcast storm, and avoid low energy nodes to participate in the establishment of the path. The results of simulation show that NS-AOMDV can effectively improve the networks' packets delivery rate, through put and normalized routing overhead in the situation of dynamic network topology and heavy load.
\end{abstract}

Keywords: Ad Hoc; AOMDV; Node State; Path Weight; Packet Delay Forwarding; Energy Threshold

\section{Introduction}

Mobile ad hoc network is a self-organized and special wireless communication network, which is made up of some mobile nodes by using distributed protocols. Since each node in ad hoc network has the function of the host and router, it has the characteristics of flexibility and convenience in deployment. In the situation of no fixed network infrastructure, mobile ad hoc network can communicate with each other by using multi-hop way, and provide the convenience to some special occasions such as medical, meeting, military and so on. Routing protocol [1] plays an important role in the communication between nodes. And the research of it has become a hot spot.

This paper discusses the existing defects when the AOMDV [2,3] selects the main path. We introduce NSAOMDV, a protocol based on node state that can effectively improve the performance of AOMDV.

The rest of the paper is organized as follows. Section 2 describes the related work. In Section 3, we make some network model assumptions. The design of NS-AOMDV is showed in Section 4, and its performance is evaluated and compared with AOMDV and AODV [4] in Section 5. At last, we make a short conclusion in Section 6.

\section{Related Work}

The main idea in AOMDV is to compute multiple paths during route discovery procedure for contending link failure [5]. When AOMDV builds multiple paths, it will select the main path for data transmission which is based on the time of routing establishment. The earliest one will be regarded the best one, and only when the main path is down other paths can be effective. In fact, a large number of studies indicate that the aforementioned scheme is not necessarily the best path. Mobile nodes, which usually due to residual energy are too low or under heavy load and other factors, seriously affect the performance of the network. In order to improve the performance, we propose the novel NS-AOMDV protocol based on existing AOMDV. First, we consider the rate of node residual energy and idle buffer queue as the weight of node. Second, in route discovery process, the routing update rules calculate the node weight of each path and sort the path weight by descending value of path weight in route list, and we choose the path which has the largest path weight to transmit data packets. At the same time, the protocol uses the technology of RREQ delay forwarding [6] and energy threshold to ease network congestion, limit the RREQ broadcast storm, and avoid low energy nodes to participate in the establishment of the path.

\section{Network Model Assumptions}

In the process of designing routing protocols, we make 
some network model assumptions:

- We assume the ad hoc network is an undirected graph $G=<N, L>$, where $N$ is the number of nodes in the network, and $L$ represents the number of link. In this network, each node owns the ability of receiving and forwarding data.

- Every node is made up of some network components. These components include a network interface, the MAC layer, interface queue, link layer and the module for node receiving information and processing information, etc. Mobile node information can got through these artifacts.

- In the network, each mobile node is peer-to-peer, shares radio channel, and uses the IEEE 802.11 protocol in MAC layer.

\section{NS-AOMDV Design}

\subsection{The State Parameters of The Node}

\section{1) Residual Energy Rate}

This paper first defines residual energy rate $e_{i}(t)$, which refers to the residual energy level of the node $n$ at a certain time of $t$. The formula is shown as follows:

$$
e_{i}(t)=\frac{E_{i}(t)}{E_{\text {initial }}} .
$$

Where, $e_{i}(t)$ is the residual energy of the node at time $t$, and $E_{\text {initial }}$ is the initial energy of it. We can easily find that $e_{i}(t)$ indicates one node's level of energy consumption. At the same time, this parameter also can indirectly reflect the location of a node in the network. In the network, each node produces energy consumption due to sensing the signal of the neighbor nodes around. Then excessive power consumption means large node density of one node in the case of common communication service. As a result, it can be concluded that it's under heavy load in the process of communication.

2) The Idle Rate of Buffer Queue

The idle rate of buffer queue is expressed by the formula below:

$$
l_{i}(t)=\frac{L_{\max }-L_{i}(t)}{L_{\max }} .
$$

Where, $L_{i}(t)$ is defined as the length of the buffer queue at time $t . \quad L_{\max }$ means the maximum length of the buffer queue. This formula reflects the congestion status of the network. The smaller available buffer queue length means more data packets need to be processed and worse network congestion.

In ad hoc networks, a mobile node can be considered as a consist of the network interface, the MAC layer, interface queue, the link layer and the modules of receiving and processing information, as shown in Figure 1. It shows the MAC layer and the link layer; when a node in

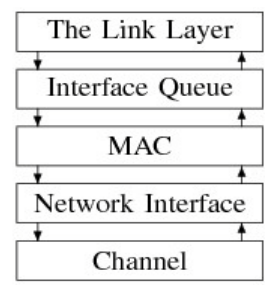

Figure 1. Diagram for mobile node structure.

the transmitted data packet, the data stream is usually required in the node interface queue filter delete processing. Therefore, we get the idle rate of buffer queue by calculating the length of the interface queue, namely we utilize the bottom of the interface queue information to reflect the network status.

3) Node Weight

To some extent, two state parameters above reflect the status of the network. In this paper, we will take both of them into consideration, and propose a new definition which is called Node Weight $(N W)$. The formula is shown as follows:

$$
N W_{i}(t)=\alpha e_{i}(t)+\beta l_{i}(t) .
$$

Where, $\alpha+\beta=1(0 \leq \alpha \leq 1,0 \leq \beta \leq 1)$, if $\alpha>\beta$, the residual energy rate is the main consideration. If $\alpha<\beta$, we contend the idle rate of buffer queue as the main influencing factor.

\subsection{Selecting The Main Path}

First, we define Path Weight $(P W)$ as the least $N W$ of all nodes on a path. Its computation formula is as follows:

$$
P W_{i}(t)=\left\{\min \left(N W_{i}(t)\right) \mid i \in N O D E\right\} .
$$

$P W_{i}(t)$ is the path weight at time $t$, and NODE is the set of nodes on a path. Because we need to take the level of every path into consideration when we select the main path, it includes two important steps: updating path weight and selecting the main path.

1) Updating Path Weight

Updating node weight occurs in the time of routing updating. Meanwhile, we can get path weight of every path. NS-AOMDV is similar to AOMDV, and its main difference is that NS-AOMDV needs to update node weight in the step of updating route.

2) Selecting The Main Path

The main idea in AOMDV is to compute multiple paths during route discovery procedure for contending link failure. When AOMDV creates multiple paths between source node and destination node, only the path based on some metric is chosen for data transmission.

In other words, the path which first reaches the destination node is chosen for the primary path and the other paths will become alternate ones. In this way, we can quickly create a path for data transmission. However, we 
ignore the state of the node's own level and other factors. Nodes, which own the low level of residual energy and heavy load, may exist in the primary path. If so, this path is very likely to be disconnected because of energy depletion.

Compared to AOMDV, NS-AOMDV firstly utilizes the forward path on which the first RREP packet arrives at source node earliest for data transmission. When multiple RREP packets arrive at the source node, it will utilize the path which owns the largest $P W$ recorded in the routing table for data transmission. Because $P W s$ are in descending order in route table, we can always select the path of largest $P W$ every time. The formula is as follows:

$$
\text { route }_{\text {list }}=\max \left\{P W_{p}(t) \mid p \in P A T H\right\} .
$$

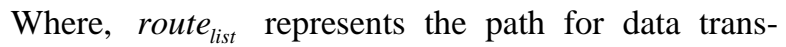
mission and PATH is the set of paths in routing table.

\subsection{Technology of RREQ Delay Forwarding}

In the route discovery procedure, we can also control those intermediate nodes with heavy load to delay forwarding RREQ packet, based on the level of $N W$. Its main goal is to allow a node with lighter load to be quickly involved in setting up the path. Its main purpose is to make a lighter load node to participate in the establishment of the path quickly. Also the node with heavy load can participate in the establishment of the path again when the network status improves. In this way, network traffic is balanced and network congestion is avoided efficiently. The paths will be relatively independent at the same time. We get the RREQ delay forwarding time based on the formula below:

$$
\operatorname{delay}_{i}(t)=\left\{\begin{array}{ll}
T_{c}, & N W_{i}(t)>0.5 \\
\frac{T_{c}}{N W_{i}(t)}, & N W_{i}(t) \leq 0.5
\end{array} .\right.
$$

Where, $\operatorname{delay}_{i}(t)$ is the time for intermediate node $i$ forwarding RREQ packet. In AOMDV, $T_{c}$ is the time that set for forwarding the RREQ packet by default. If $N W_{i}(t)>0.5$, it reflects the node owns light load. So if $N W_{i}(t)<0.5$, it means the node is busy now, it should delay forward this RREQ packet.

\subsection{Technology of Energy Threshold}

It's very dangerous while the intermediate node owns low level of energy and it also needs to forward RREQ packet simultaneously. If you accidentally use it to establish one route, it will easily lead to the emergence of network segmentation. In order to avoid selecting this kind of nodes involved in the route setup, we set up an energy threshold $E_{\text {threshold }}$ to exclude them. The energy threshold size setting refers to the Ref. [7] setting method.
Ref. [7] points out that one node's energy level is classified as a "discarded" level, and its survival time will be estimated less than 10s when the residual energy of it is less than $10 \%$ of the initial. We propose to set the energy threshold $20 \%$ based on this. In the process of intermediate node processing RREQ packet, only when its own residual energy rate is larger than $E_{\text {threshold }}$, will it forwards the RREQ packet.

\section{Performance Evaluation}

To evaluate the performance of NS-AOMDV, we compare it with AODV and AOMDV by using NS2.34. In the process of simulation, we assume every protocol shares the same model and node configuration. Their initial parameters are shown in Table 1. Also we assume $\alpha=\beta=$ 0.5 . We evaluate the performance of NS-AOMDV through four metrics below:

- Packet delivery rate

- Throughput

- The normalized routing overhead

- The average end-to-end delay

\subsection{Simulation Scenario 1: Varying Mobility}

We set the speed of sending packets 1 packet/s. Pause time for the node is 10 seconds. The maximum number of connections between the nodes is 20 . The maximum moving speed of the node changes in $5 \mathrm{~m} / \mathrm{s}, 10 \mathrm{~m} / \mathrm{s}, 15$ $\mathrm{m} / \mathrm{s}, 20 \mathrm{~m} / \mathrm{s}, 25 \mathrm{~m} / \mathrm{s}, 30 \mathrm{~m} / \mathrm{s}$.

Figure 2 plots packet delivery rate against the maximum moving speed. The graph demonstrates packet delivery rate of the three protocols are significantly reduced with the increase of node maximum speed. But NSAOMDV has a higher packet delivery rate than the other two. The reasons for NS-AOMDV's better performance are shown as follows:

Table 1. Initial parameters for node configuration.

\begin{tabular}{cc}
\hline Parameter & Value \\
\hline Dimensions & $1000 \mathrm{~m} \times 1000 \mathrm{~m}$ \\
Number of nodes & 30 \\
Source type & CBR \\
Antenna Type & Omnidirectional \\
Spread type & TwoRayGround \\
Wireless channel capacity & $2 \mathrm{Mb} / \mathrm{s}$ \\
Communication radius & $250 \mathrm{~m}$ \\
Packet size & $512 \mathrm{bytes}$ \\
Initial Energy & $60 \mathrm{~J}$ \\
Transmission power & $1.3 \mathrm{~W}$ \\
Reception power & $0.8 \mathrm{~W}$ \\
Buffer size & 50 \\
MAC Layer & IEEE802.11b DCF \\
Transport Layer & UDP \\
Simulation time & $300 \mathrm{~s}$
\end{tabular}




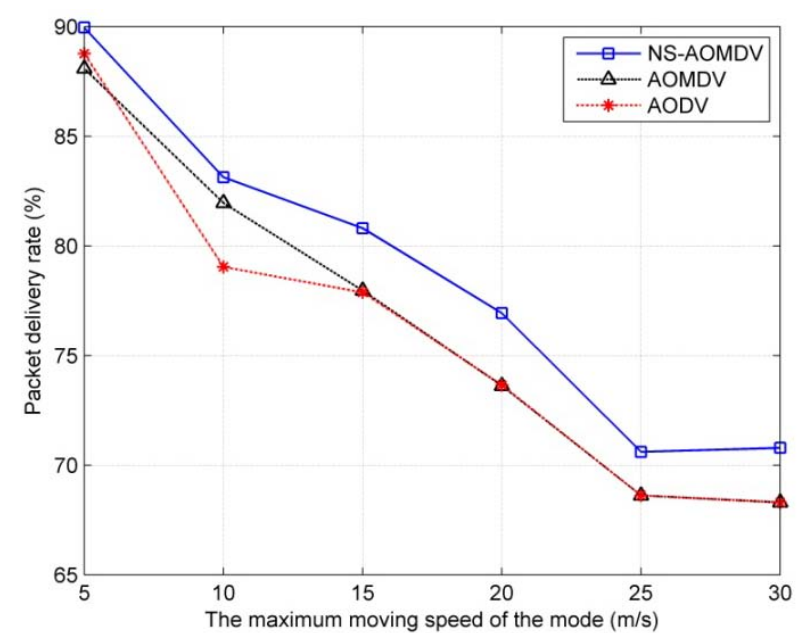

Figure 2. Packet delivery rate as the changes of the maximum moving speed of the node.

- Comprehensive consideration of residual energy rate and idle rate of buffer queue in selecting the main path.

- Setting energy threshold avoids the nodes with lower energy participating in the construction of the path.

These measures ensure the main path more robust and data transmission more reliable.

Figure 3 reflects the network throughput varies with the changes of speed. When the mobile nodes speed up, throughput of the three agreements declines significantly. Because NS-AOMDV employs the technology of RREQ delay forwarding, it limits the broadcast storm of RREQ, eases the network congestion and balances network traffic. It also takes the length of buffer queue in the process of selecting the main path. All aforementioned schemes causes higher throughput.

Figure 4 shows the normalized routing overhead increases with the increase of maximum moving speed. Due to path failure, AODV needs to re-route discovery process, which corresponding increases routing overhead simultaneously. In the other two protocols, NS-AOMDV owns higher normalized routing overhead at the speed of 20 $\mathrm{m} / \mathrm{s}$. Overall, NS-AOMDV is better than AOMDV. The main reason is that NS-AOMDV sets the energy threshold, forces some node with low energy not to forward RREQ packets and reduces the amount of control packets sent. On the other hand, RREQ delay forwarding improves the ratio for node processing RREQ packets, optimizes the forwarding mechanism, and avoids some unnecessary routing overheads.

Figure 5 demonstrates that the average end-to-end delay has a tendency to increase with the acceleration of the maximum speed of the mobile node. i.e., the instability of the network topology causes that time for data transmission and processing increases. Because AODV has no

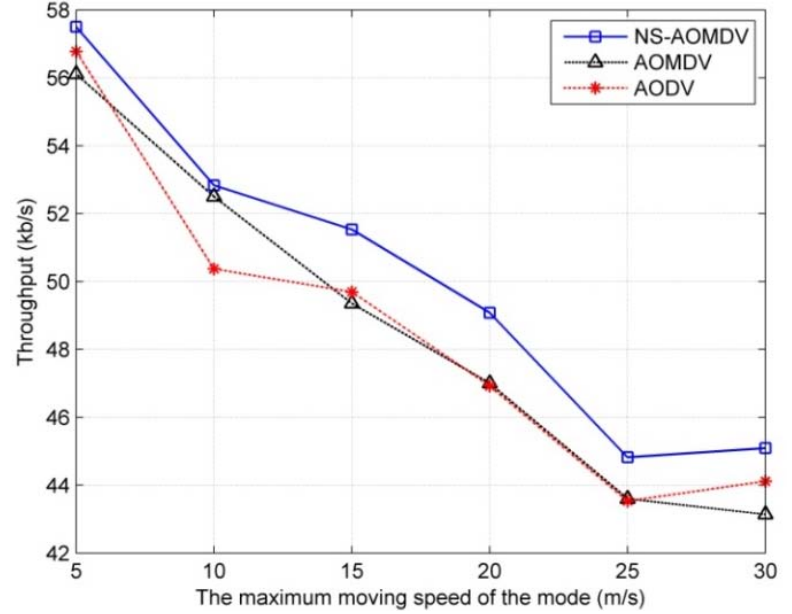

Figure 3. Throughput as the changes of the maximum moving speed of the node.

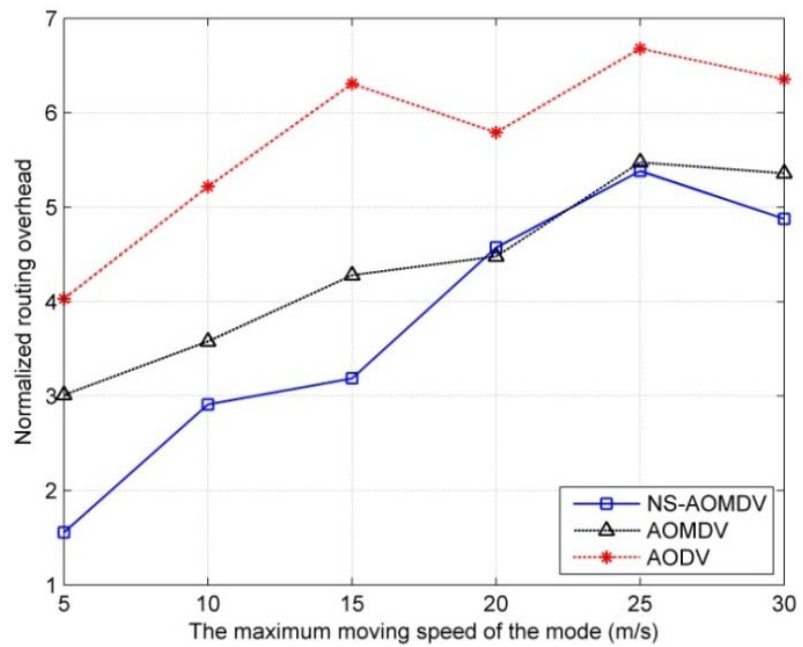

Figure 4. Normalized routing overhead as the changes of the maximum moving speed of the node.

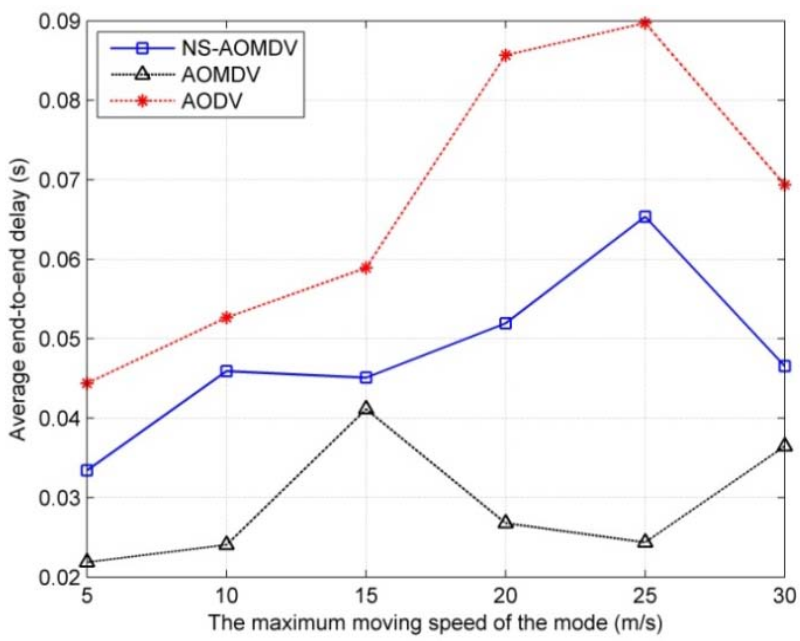

Figure 5. Average end-to-end delay as the changes of the maximum moving speed of the node. 
alternate path, it needs to re-route discovery process and consume a certain period of time when the primary link is broken. In multi-path protocols, there are more advantages in this aspect. The average end-to-end delay of NS-AOMDV is larger than AOMDV. On the one hand, in the process of forwarding RREQ packet, NS-AOMDV needs delay forwarding according to the state of the node. Therefore it spends extra time. On the other hand, in selecting a path for data transmission, NS-AOMDV focuses on the reliability, while AOMDV focuses on the time. So NS-AOMDV makes a certain sacrifice in the average end-to-end delay.

\subsection{Simulation Scenario 2: Varying Pause Time}

We set the speed of sending packets 1 packet/s. The maximum number of connections between the nodes is 20 . The maximum moving speed of the node is $10 \mathrm{~m} / \mathrm{s}$. The pause time of the node changes in 0 second, 20 seconds, 40 seconds, 60 seconds, 80 seconds, 100 seconds.

Figure 6 shows packet delivery rate is low when network topology changes rapidly. Packets delivery rate increases with the augment of node's pause time. Since the introduction of node weight, NS-AOMDV ensures the selected path robust enough. Especially when the network tends to be stable, NS-AOMDV shows better performance than the others.

Figure 7 demonstrates that throughput augments significantly when the pause time increases dramatically. The introduction of node weight and technology of RREQ delay forwarding, which balances the utilization of various nodes in the network, improves the performance of throughput.

Figure 8 shows the changes in normalized routing overhead. There is a decreasing trend in the normalized routing overhead of the three protocols. This illustrates that with the increase of pause time, the rate for the destination node successfully accepting the request packet improves. With the changes of pause time, the normalized routing overhead changes slightly, which shows the performance of the three protocols on routing overhead is relatively stable. Similarly, AODV's bigger routing overhead is mainly caused by several route discovery processes, and the local repair mechanism also requires a certain overhead. The setting of energy threshold decreases the control packets sent by low-energy node. At the same time, the delay forwarding RREQ enhances the efficiency for node processing route request packet. It makes the NS-AOMDV protocol performs better than AOMDV in this aspect.

Figure 9 shows that when the node pause time increases, the end-to-end delay of the network tends to decrease, but brings in some volatility due to the change of node mobility. It also tells us that AODV has a large gap with the other two. The main reason for the gap is re-

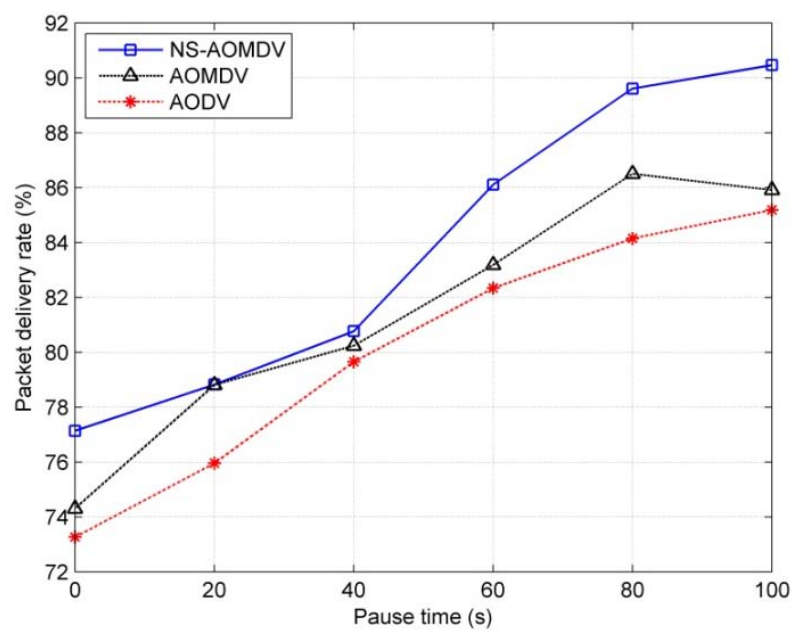

Figure 6. Packet delivery rate as the changes of pause time.

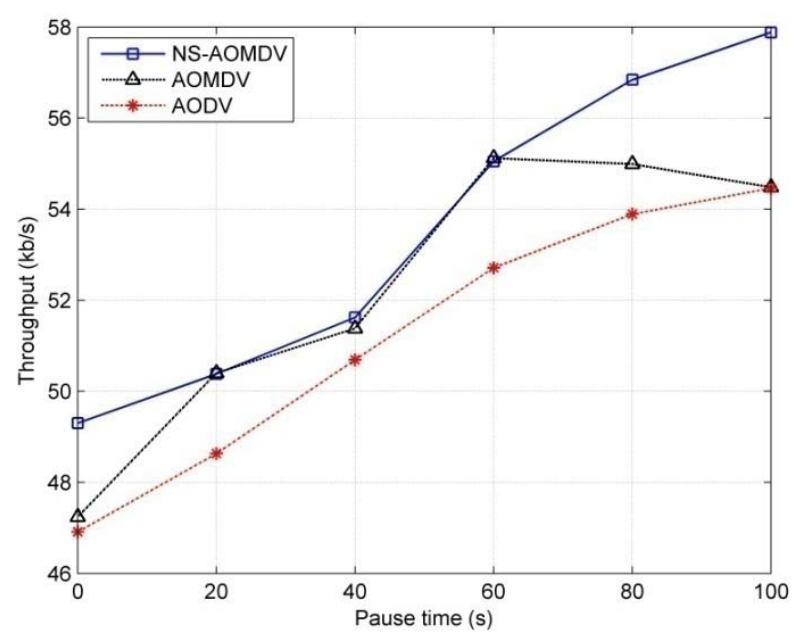

Figure 7. Throughput as the changes of pause time.

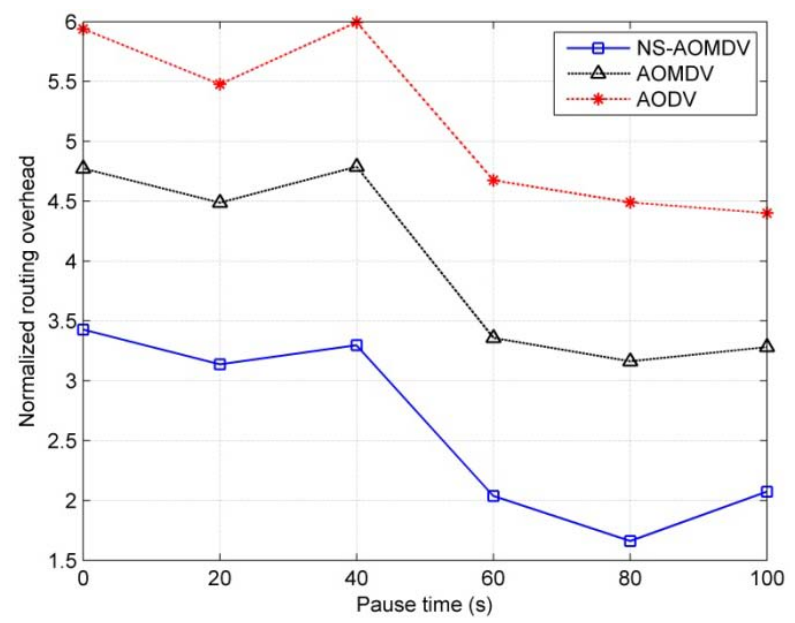

Figure 8. Normalized routing overhead as the changes of pause time.

routing discovery process takes time, and the frequency of route discovery is faster than multi-path protocols. For 


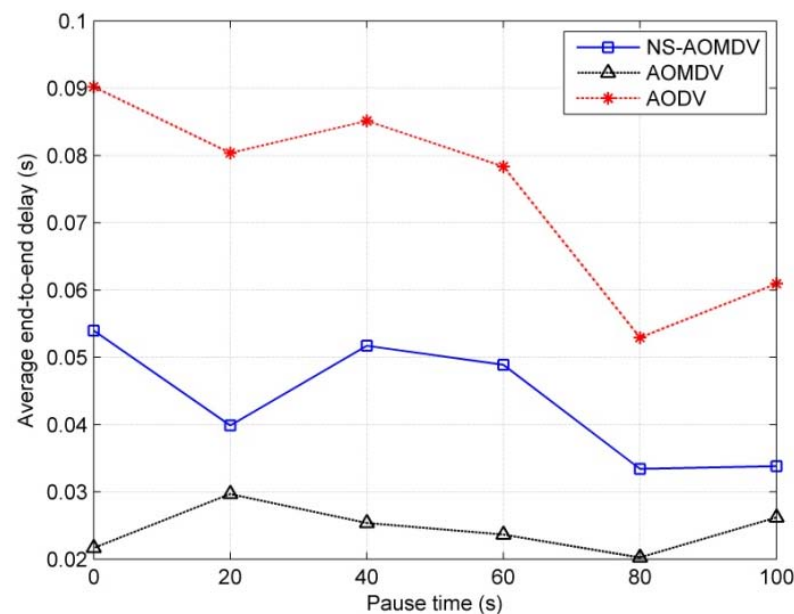

Figure 9. Average end-to-end delay as the changes of pause time.

two types of multi-path routing protocol, NS-AOMDV's end-to-end delay is still longer than AOMDV. This further illustrates delay forwarding RREQ and selecting the main path need some extra time.

From the simulation results of two scenarios, we can conclude that most performances of the network will get different degrees of improvement when network topology tends to be flattened. As for overall performance, AODV performs worse than AOMDV and NS-AOMDV especially in time delay and normalized routing overhead. For two multi-path protocols, NS-AOMDV is generally better than AOMDV.

\section{Conclusion}

In this paper, a novel multipath distance vector routing protocol, NS-AOMDV, for MANETs is proposed to improve some performances of present AOMDV. In the process of building transmission path, we synthetically consider the residual energy rate and the idle rate of buffer queue. And we introduce the technology of RREQ delay forwarding and energy threshold in route discovery. Also we update the information of the nodes on the path, and choose the path of maximum node weight for data transmission.

\section{REFERENCES}

[1] Z. Chen, L. Guan, X. Wang and X. Fan, "Ad hoc On-demand Multipath Distance Vector routing with Backup Route Update Mechanism,” IEEE 14th International Conference on High Performance Computing and Communications, 2012, pp. 908-913.

[2] K. Marina and R. Samir, "On-Demand Multipath Distance Vector Routing in Ad Hoc Networks,” International Conference on Network Protocols, 2001, pp. 14-23.

[3] K. Marina and R. Samir, "Ad Hoc on-Demand Multipath Distance Vector Routing,” Wireless Communications and Mobile Computing, 2006, pp. 969-988. http://dx.doi.org/10.1002/wcm.432

[4] E. Charles, M. Elizabeth, “Ad-Hoc on Demand Distance Vector Routing," The IEEE Workshop on Mobile Computing Systems and Applications, New Orleans, 1999, pp. 90-100.

[5] X. Li, S. Zhi and S. Xin, “Ad-Hoc Multipath Routing Protocol Based on Load Balance and Location Information," Wireless Communications \& Signal Processing (WCSP), 2009, pp. 1-4.

[6] M. Tekaya, N. Tabbane and S. Tabbane, "Multipath Routing Mechanism with Load Balancing in Ad Hoc Network," International Conference on Computer Engineering and Systems (ICCES), 2010, pp. 67-72.

[7] S. Getsy, P. Neelavathy and D. Sridharan, "Energy Efficient Ad Hoc On Demand Multipath Distance Vector Routing Protocol,” International Journal of Recent Trends in Engineering, 2009, pp. 10-12 